\title{
ON THE COMPOUND GENERALIZED POISSON DISTRIBUTIONS
}

\author{
By R.S. Ambagaspitiya AND N. BaLAKRishnaN \\ University of Calgary - McMaster University
}

\begin{abstract}
GoOvaerts and KaAs (1991) present a recursive scheme, involving Panjer's recursion, to compute the compound generalized Poisson distribution (CGPD). In the present paper, we study the CGPD in detail. First, we express the generating functions in terms of Lambert's $W$ function. An integral equation is derived for the pdf of CGPD, when the claim severities are absolutely continuous, from the basic principles. Also we derive the asymptotic formula for CGPD when the distribution of claim severity satisfies certain conditions. Then we present a recursive formula somewhat different and easier to implement than the recursive scheme of GoovAERTS and KAAS (1991), when the distribution of claim severity follows an arithmetic distribution, which can be used to evaluate the CGPD. We illustrate the usage of this formula with a numerical example.
\end{abstract}

\section{KEYWORDS}

Compound generalized Poisson distributions; moments; integral equations; recursive equation; tail behaviour.

\section{INTRODUCTION}

Modelling the claim frequency data is one of the most important areas in risk theory. Traditionally, the Poisson distribution, when the mean number of claims is equal to its variance, and the negative binomial distribution, when the variance of the number of claims exceeds its mean, have been used because of their convenient mathematical properties. Several authors including GossiauX and LEMAIRE (1981), SEAL (1982) and WILLMOT (1987) have considered alternatives to Poisson and negative binomial distributions for this purpose. Consul (1990) has compared the Generalized Poisson distribution (GPD) suggested by ConSUL and JAIN (1973) with several well known distributions and concluded that GPD is a plausible model for claim frequency data. GoOvaERTS and KAAS (1991) presented a recursive scheme to compute the total claim distribution under the assumptions that the claims are independently and identically distributed integer random variables with the GPD claim frequency.

In this paper, we discuss the compound generalized Poisson distribution (CGPD) in detail and derive a somewhat easy to programmable recursive relation than one given by GoOvaerTs and KaAs (1991). In Section 2, we present a brief summary 
of the properties of the generalized Poisson distribution. In Section 3, we express the generating functions of CGPD in terms of Lambert's $W$ function and illustrate the derivation of moments. In Section 4, we present an integral equation similar to Volterra's integral equation of second kind for the density function of CGPD when the distribution of claim severity is absolutely continuous. In addition, we discuss the tail behaviour of CGPD when the claim severity is non arithmetic. In Section 5, we present a recursive formula for the probability function of CGPD when the distribution of claim severity is arithmetic. We illustrate the usage of this formula through an example.

\section{Generalized Poisson distribution (GPD)}

CONSUL and JAIN (1973) proposed a new generalization of the discrete Poisson distribution which was modified by Consul and SHOukri (1985) to: A discrete random variable $N$ is said to have a generalized Poisson distribution (GPD) if its probability mass function is given by

(2.1) $\operatorname{Pr}(N=n)=p_{n}(\lambda, \theta)= \begin{cases}\lambda(\lambda+n \theta)^{n-1} \frac{\exp (-\lambda-n \theta)}{n !} & \text { for } n=0,1,2 \ldots \\ 0 & \text { for } n>m \text { when } \theta<0\end{cases}$

and zero otherwise, where $\lambda>0, \max (-1,-\lambda / m) \leq \theta<1$ and $m(\geq 4)$ is the largest positive integer for which $\lambda+\theta m>0$ when $\theta$ is negative. This generalization of the Poisson probability model in the sense that is probability generating function (pgf) is given by the Lagrange expansion of any pgf under a suitable transformation (Consul and SHENTON (1972)). The GPD reduces to the Poisson distribution when $\theta=0$ and it possesses the twin properties of over-dispersion and under-dispersion according as $\theta>0$ or $\theta<0$. The GPD gets truncated for negative values of $\theta$ but the truncation error is always less than $0.07 \%$. A recent book by Consul (1989) discusses various properties, inference and numerous applications of this model in biology, ecology, and other disciplines. For simplicity, from here on we assume the parameter $\theta>0$. AMBAGASPITIYA and BALAKRISHNAN (1993) has recently expressed the moment generating function $M_{N}(t)$ and the probability generating function of the GPD in terms of Lambert's $W$ function when $\theta>0$ as follows :

$$
\begin{aligned}
& M_{N}(t)=\exp \left\{-\frac{\lambda}{\theta}[W(-\theta \exp (-\theta+t))+\theta]\right\} \\
& P_{N}(z)=\exp \left\{-\frac{\lambda}{\theta}[W(-\theta z \exp (-\theta))+\theta]\right\}
\end{aligned}
$$

where $W$ is the Lambert's $W$ function defined as

$$
W(x) \exp (W(x))=x
$$

For more details about Lambert's $W$ function see CoRLESs et al. (1994). 


\subsection{Central moments of GPD}

We can obtain the central moments of GPD by differentiating (2.2) with respect to $t$ as illustrated by AMBAGASPITIYA and BALAKRISHNAN (1993), or from the basic principles as described by CoNSUL (1989), or by using the method suggested by GOOVAERTS and KAAS (1991). The resulting expressions for first four central moments are as follows:

$$
\begin{aligned}
& \mu_{1}=\lambda M \\
& \mu_{2}=\lambda M^{3} \\
& \mu_{3}=\lambda(3 M-2) M^{4} \\
& \mu_{4}=3 \lambda^{2} M^{6}+\lambda\left(15 M^{2}-20 M+6\right) M^{5}
\end{aligned}
$$

where $M=(1-\theta)^{-1}$.

\subsection{Maximum likelihood estimators of $\lambda$ and $\theta$}

Let a random sample of $n$ items be taken from the GPD model and let $x_{1}, x_{2}, \ldots, x_{n}$ be their corresponding values. If the sample values are classified into class frequencies and $n_{i}$ denotes the frequency of the $i$ th class $\left(n_{i}=\#\left\{x_{j}: 1 \leq j \leq n, x_{j}=i\right\}\right)$, the ML estimate $\hat{\theta}$ as described in Consul and SHOUKRI (1984) is given by the unique root of $\theta$ given by the equation

$$
\sum_{i=0}^{k} n_{i} \frac{i(i-1)}{\bar{x}+(i-\bar{x}) \theta}-n \bar{x}=0
$$

where $k(\leq 2)$ is the number of classes, $n=\sum_{i=1}^{k} n_{i}$ and $\bar{x}$ is the sample mean. Note that (2.5) does not give a value for $\theta$ when $k=0$ or 1 . The ML estimate $\hat{\lambda}$ is then given by

$$
\hat{\lambda}=\bar{x}(1-\hat{\theta})
$$

\subsection{Tail behaviour of GPD}

Lemma 2.1: For fixed $\lambda, \theta$ and $n \rightarrow \infty$

$$
\operatorname{Pr}(N=n) \approx \frac{\lambda}{\theta \sqrt{2 \pi}} \exp \left(-\lambda+\frac{\lambda}{\theta}\right) n^{-3 / 2} \cdot(\theta \exp (1-\theta))^{n}
$$

\section{Proof :}

For large $n$, using the Stirling approximation to $n$ ! we can write the pmf in (2.1) as

$$
\operatorname{Pr}(N=n) \approx \frac{\lambda(\lambda+n \theta)^{n-1} \exp (-\lambda-n \theta)}{\sqrt{2 \pi} n^{n+1 / 2} \exp \left(-n+\frac{\theta_{1}}{12 n}\right)}
$$


where $\theta_{1}=\theta_{1}(n)$ satisfies $0<\theta_{1}<1$. After some rearrangement, we have

$$
\operatorname{Pr}(N=n) \approx\left\{\frac{\lambda}{\theta \sqrt{2 \pi}}\left[1+\frac{\lambda}{\theta n}\right]^{n-1} \exp \left(-\lambda-\frac{\theta_{1}}{12 n}\right)\right\} n^{-3 / 2}(\theta \exp (1-\theta))^{n}
$$

Note that the term inside the \{\} tends to the required constant as $n \rightarrow \infty$ and hence the proof.

\section{COMPOUnd GENERAlized PoISSON Distribution (CGPD)}

Let $N$ denote the number of claims produced by a portfolio of policies in a given time period. Let $X_{i}$ denote the amount of the $i$ th claim. Then

$$
S=X_{1}+X_{2}+\ldots+X_{N}
$$

represents the aggregate claims generated by the portfolio for the period under study. In order to make the model tractable, two fundamental assumptions are made in risk theory and they are

1. $X_{1}, X_{2}, \ldots$ are identically distributed random variables with the distribution function $F(x)$.

2. The random variables $N, X_{1}, X_{2}, \ldots$ are mutually independent.

When a GPD is chosen for $N$, the distribution of $S$ is called a compund generalized Poisson distribution. In terms of the convolution operation, we can write the distribution function of $S$ as :

$$
F_{S}(x)=\sum_{n=0}^{\infty} F^{* n}(x) \lambda(\lambda+n \theta)^{n-1} \frac{\exp (-\lambda-n \theta)}{n !}
$$

The moment generating function of $S$ is given by

$$
M_{S}(t)=M_{N}\left(\log M_{X}(t)\right),
$$

where $M_{N}(t)$ is the moment generating function (mgf) of the GPD and $M_{X}(t)$ is the mgf of the claim amount distribution. By using the expression given in (2.2), we can write the mgf of $S$ as

$$
M_{S}(t)=\exp \left\{-\frac{\lambda}{\theta}\left[W\left(-\theta \exp (-\theta) M_{X}(t)\right)+\theta\right]\right\} .
$$

Similarly, the probability generating function (pgf) of $S$, when the distribution of claim severity is arithmetic, can be written as

$$
P_{S}(z)=\exp \left\{-\frac{\lambda}{\theta}\left[W\left(-\theta \exp (-\theta) P_{X}(z)\right)+\theta\right]\right\},
$$

where $P_{X}(z)$ is the pgf of claim amount distribution. 


\subsection{Central moments of $S$}

The moments of $S$ can be obtained by directly differentiating the mgf of $S$ given in (3.3). For this differentiation, one may use the following identity, involving Lamberts $W$ functions :

$$
\frac{d W(x)}{d x}=\frac{W(x)}{x(1+W(x))} .
$$

After some lengthy algebra, we obtain the following expressions for the first three central moment of $S$ :

$$
\begin{array}{ll}
E(S) & =\lambda p_{1} M \\
\operatorname{Var}(S) & =\lambda p_{2} M^{3}+\lambda\left(p_{2}-p_{1}^{2}\right) M \\
E\left((S-E(S))^{3}\right) & =\lambda(3 M-2) p_{1}^{3} M^{4}+3 \lambda p_{1}\left(p_{2}-p_{1}^{2}\right) M^{3}+\left(p_{3}-3 p_{2} p_{1}+2 p_{1}^{3}\right) \lambda M
\end{array}
$$

where $M=(1-\theta)^{-1}$ and $p_{i}, i=1,2,3$ are the $i$ th non-central moments of claim severity.

\section{PROPERTIES OF CGPD: ABSOLUTELY CONTINUOUS SEVERITIES}

Theorem 4.1: If the claim sizes are absolutely continuous with pdf $f(x)$ for $x>0$, then the pdf $g(\lambda, \theta ; x)$ of CGPD satisfy the integral equation

$$
g(\lambda, \theta ; x)=p_{1}(\lambda, \theta) f(x)+\frac{\lambda}{\lambda+\theta} \int_{0}^{x}\left(\theta+\lambda \frac{y}{x}\right) g(\lambda+\theta, \theta ; x-y) f(y) d y
$$

where $p_{1}(\lambda, \theta)=\operatorname{Pr}(N=1)$ in the GPD with parameters $\lambda$ and $\theta$.

\section{Proof :}

Consider

$$
\begin{aligned}
g(\lambda, \theta ; x) & =\sum_{i=1}^{\infty} p_{i}(\lambda, \theta) f^{* i}(x) \\
& =p_{1}(\lambda, \theta) f(x)+\sum_{i=2}^{\infty} p_{i}(\lambda, \theta) f^{* i}(x)
\end{aligned}
$$

By using the following identity of GPD,

$$
p_{i}(\lambda, \theta)=\frac{\lambda}{\lambda+\theta}\left(\theta p_{i-1}(\lambda+\theta, \theta)+\frac{\lambda}{i} p_{i-1}(\lambda+\theta, \theta)\right) \quad i=1,2, \ldots
$$

we have

$$
\begin{aligned}
\sum_{i=2}^{\infty} p_{i}(\lambda, \theta) f^{* i}(x)= & \frac{\lambda}{\lambda+\theta}\left(\theta \sum_{i=2}^{\infty} p_{i-1}(\lambda+\theta, \theta) f^{* i}(x)+\right. \\
& \left.+\lambda \sum_{i=2}^{\infty} \frac{p_{i-1}(\lambda+\theta, \theta)}{i} f^{* i}(x)\right)
\end{aligned}
$$


Using the identities

$$
f^{* i}(x)=\int_{0}^{x} f^{*(i-1)}(x-y) f(y) d y
$$

and

$$
\frac{f^{* i}(x)}{i}=\int_{0}^{x} \frac{y}{x} f^{*(i-1)}(x-y) f(y) d y
$$

we have

$$
\begin{aligned}
\sum_{i=2}^{\infty} p_{i}(\lambda, \theta) f^{* i}(x)= & \frac{\lambda}{\lambda+\theta}\left(\theta \sum_{i=2}^{\infty} p_{i-1}(\lambda+\theta, \theta) \int_{0}^{x} f^{*^{(i-1)}}(x-y) f(y) d y\right. \\
& \left.+\lambda \sum_{i=2}^{\infty} p_{i-1}(\lambda+\theta, \theta) \int_{0}^{x} \frac{y}{x} f^{*(i-1)}(x-y) f(y) d y\right)
\end{aligned}
$$

By interchanging the order of summation and the integration and realizing the fact

$$
\begin{aligned}
\sum_{i=2}^{\infty} p_{i-1}(\lambda+\theta, \theta) f^{*(i-1)}(x-y) & =\sum_{i=1}^{\infty} p_{i}(\lambda+\theta, \theta) f^{* i}(x-y) \\
& =g(\lambda+\theta, \theta ; x-y)
\end{aligned}
$$

we have

$$
\sum_{i=2}^{\infty} p_{i}(\lambda, \theta) f^{* i}(x)=\frac{\lambda}{\lambda+\theta} \int_{0}^{x}\left(\theta+\lambda \frac{y}{x}\right) g(\lambda+\theta, x-y) f(y) d y
$$

Substitution of (4.11) in (4.3) yields the required result and hence the theorem.

One has to solve the integral equation (4.1) numerically. Although, there are many algorithms and implementations available to solve Volterra integral equations of the second kind, one has to modify them to solve (4.1). We are currently investigating the problem of finding the best algorithm and we hope to report this finding in a future article.

\subsection{Tail behaviour of CGPD}

Theorem 4.2: If there exists a number $k>0$ satisfying

$$
\frac{\exp (\theta)}{e \theta}=\mathcal{L}_{X}(-\kappa)
$$


for $X$ non-arithmetic and if $-L_{X}^{\prime}(-k)<\infty$, then

$$
1-F_{S}(x) \sim C x^{-3 / 2} \exp (-\kappa x),
$$

where $C$ is given by

$$
C=\frac{\lambda}{\theta \sqrt{2 \pi}} \exp \left(-\lambda+\frac{\lambda}{\theta}\right) \sqrt{\kappa\left[-\frac{\exp (\theta-1)}{\theta} \mathcal{L}_{x}^{\prime}(-\kappa)\right]}
$$

\section{Proof :}

The Proof of this theorem directly follows from the Lemma 2.1 and from the theorem of Embrechts, Maejima, and Teugels (1982).

\section{PROPERTIES OF CGPD: ARITHMETIC SEVERITIES}

Theorem 5.1: If the claim sizes are random variables on the positive integers with probability mass function $f(x)=\operatorname{Pr}(X=x), x=0,1,2, \ldots$, then the probability mass function $g(\lambda, \theta ; x)$ of CGPD satisfies the recurrence equation

$$
g(\lambda, \theta ; x)=\frac{\lambda}{\lambda+\theta} \sum_{y=1}^{x}\left(\theta+\lambda \frac{y}{x}\right) g(\lambda+\theta, \theta ; x-y) f(y) \text {. }
$$

\section{Proof :}

This theorem can be proved following the same line of reasoning as Theorem 4.1 or the standard proof of Panjer's recursion (see Theorem 6.6.1 and Corollary 6.6.1 in Panjer and Willmot (1992)).

A result analogue to Theorem 4.2 can be established for discrete severity case using Lemma 2.1 and the theorem given in WILMOT (1989).

\subsection{Recursive evaluation}

The recursive formula given in (5.1) is easily programmable and also simple to use for manual calculations. For the latter, one may use the following schematic approach :

$$
\begin{array}{lllll}
g(\lambda, \theta, 0) & g(\lambda+\theta, \theta, 0) & g(\lambda+2 \theta, \theta, 0) & g(\lambda+3 \theta, \theta, 0) & g(\lambda+4 \theta, \theta, 0) \\
g(\lambda, \theta, 1) & g(\lambda+\theta, \theta, 1) & g(\lambda+2 \theta, \theta, 1) & g(\lambda+3 \theta, \theta, 1) \\
g(\lambda, \theta, 2) & g(\lambda+\theta, \theta, 2) & g(\lambda+2 \theta, \theta, 2) & \\
g(\lambda, \theta, 3) & g(\lambda+\theta, \theta, 3) & & \\
g(\lambda, \theta, 4) & & &
\end{array}
$$


The first row of the above scheme is obtained by using the fact that $g(\lambda+i \theta, 0)=$ $p_{0}(\lambda+i \theta, \theta)=\exp (-\lambda-i \theta)$ for $i=0,1 \ldots$ To calculate the probability mass function given in the $(i, j)$ th location, one has to use the elements in $(l, j+1)$ where $l=0,1, \ldots, i-1$. Since the scheme is of an upper diagonal form, we can carry out the computations for each row starting from right to left. For example, if one wishes to compute $g(\lambda, \theta, 4)$ one may start from $g(\lambda+4 \theta, \theta, 0)$ and move along the diagonal from right to left, i.e. calculate $g(\lambda+(4-i) \theta, \theta, i), i=0,1,2,3,4$ in that order.

\section{Example :}

Suppose that $S$ has a CGPD with $\lambda=0.8, \theta=0.5$ and the distribution of individual claim amounts is as follows:

\begin{tabular}{|c|c|}
\hline$x$ & $\operatorname{Pr}(X=x)$ \\
\hline 1 & 0.25 \\
2 & 0.45 \\
3 & 0.30 \\
\hline
\end{tabular}

Then, by using the recursive method described above, the pmf of $S$ has been tabulated for $s=0(1) 59$ and these values are presented in Table 1 .

TABLE 1

THE PROBABILITY MASS FUNCTION OF $S$

\begin{tabular}{rrrrrl}
\hline \hline$s$ & $\operatorname{Pr}(S=s)$ & $s$ & $\operatorname{Pr}(S=s)$ & $s$ & $\operatorname{Pr}(S=s)$ \\
\hline 0 & .44933 & 20 & .00269 & 40 & .00017 \\
1 & .05451 & 21 & .00231 & 41 & .00015 \\
2 & .10555 & 22 & .00198 & 42 & .00013 \\
3 & .09329 & 23 & .00171 & 43 & .00012 \\
4 & .04809 & 24 & .00148 & 44 & .00010 \\
5 & .04813 & 25 & .00128 & 45 & .00009 \\
6 & .03595 & 26 & .00111 & 46 & .00008 \\
7 & .02737 & 27 & .00096 & 47 & .00007 \\
8 & .02320 & 28 & .00083 & 48 & .00006 \\
9 & .01835 & 29 & .00073 & 49 & .00006 \\
10 & .01505 & 30 & .00063 & 50 & .00005 \\
11 & .01248 & 31 & .00055 & 51 & .00004 \\
12 & .01029 & 32 & .00048 & 52 & .00004 \\
13 & .00860 & 33 & .00042 & 53 & .00003 \\
14 & .00720 & 34 & .00037 & 54 & .00003 \\
15 & .00605 & 35 & .00032 & 55 & .00003 \\
16 & .00512 & 36 & .00028 & 56 & .00002 \\
17 & .00434 & 37 & .00025 & 57 & .00002 \\
18 & .00369 & 38 & .00022 & 58 & .00002 \\
19 & .00315 & 39 & .00019 & 59 & .00002 \\
& & & & & \\
\hline
\end{tabular}




\section{ACKNOWLEDGMENTS}

The authors would like to thank both referees for their valuable comments when revising this paper. This work was supported by the Natural Siences and Engineering Research Council of Canada.

\section{REFERENCES}

AmbagaspitiYa, R.S. and Balakrishnan, N. (1993) Some remarks on Lagragian distributions, Submitted for publication.

Consul, P.C. (1989) Generalized Poisson Distributions: Properties and Applications. Marcel Dekker Inc., New York/Basel.

Consul, P.C. (1990) A model for distributions of injuries in auto-accidents. Itteilungen der Schweiz Vereinigung der Versicherungsmathematiker Heft 1, 161-168.

Consul, P.C. and Jain, G.C. (1973) A generalization of Poisson distribution. Technometrics 15, 791-799.

Consul, P.C. and Shenton, L.R. (1972) Use of Lagrange expansion for generating discrete generalized probability distributions. SIAM Journal of Applied Mathematics 23, 239-248.

Consul, P.C. and Shoukri, M. M. (1984) Maximum likelihood estimation for the generalized Poisson distribution. Communications in Statistics- Theory and Methods 10, 977-991.

Consul, P.C. and Shoukri, M. M. (1985) The generalized Poisson distribution when the sample mean is larger than the sample variance. Communications in Statistics - Simulation and Computation 14. 1533-1547.

Corless, R. M., Gonnet, G. H., Hare, D. E. G. and Jefrerey, D. J. (1994) The Lambert $W$ function. To appear in Advances in Computational Mathematics.

Embrechts, P., Maejima, M. and Teugel.s, J. (1982) Asymptotic behaviour of compound distributions. ASTIN Bulletin 15, 45-48.

Goovaerts, M.J. and KaAs, R. (1991) Evaluating compound generalized Poisson distributions recursively. ASTIN Bulletin 21, 193-197.

Gossiaux, A. and Lemaire, J. (1981) Methodes d'adjustement de distribution de sinistres. Bulletin of the Association of Swiss Actuaries 81, 87-95.

Panjer, H. H. and Willmot, G. E. (1992) Insurance Risk Models. Society of Actuaries. Seal, H. (1982) Mixed Poisson-an ideal distribution of claim numbers? Bulletin of the Association of Swiss Actuaries 82. 293-295.

Willmot, G.E. (1987) The Poisson-Inverse Gaussian distribution as an alternative to the Negative Binomial. Sandinavian Actuarial Journal, 113-127.

WILLMot, G.E. (1989) Limiting tail behaviour of some discrete compound distributions. Insurance: Mathematics and Economics 8, 175-185.

\section{R.S. AmbagaspitiYa}

Department of Mathematics and Statistis, University of Calgary, Calgary, Alberta, Canada, T2N IN4.

AND N. BALASKRISHNAN

Department of Mathematics and Statistics, McMaster University, Hamilton, Ontario, Canada, L8S 4KI. 